\title{
Uso del análisis estructural para caracterizar variables de calidad educativa en institución de primaria y secundaria venezolana
}

\author{
MILFRAN VELASQUEZ LUGO* \\ Universidad de Zulia - Venezuela \\ Recibido el 03-11-18; primera evaluación el 29-01-20; \\ segunda evaluación el 17-02-20; aceptado el 24-02-20
}

\section{Resumen}

El artículo busca caracterizar la institución educativa desde la gestión de calidad. Se parte de un diseńo de investigación acción participante (IAP), con enfoque hermenéutico interpretativo. La descripción metodológica surge del análisis estructural prospectivo, explorando la organización desde la planificación de actividades acorde a los procesos educativos, cónsonos a la misión, visión institucional; explorando si la dirección y organización responde a la estructura y lineamientos de la corporación donde pertenece la institución y reconociendo la evaluación o control de las actividades y personal. El análisis estructural permite el uso de MICMAC como herramienta prospectiva. Entre los hallazgos surgen variables claves desde la prospectiva, destacando la planificación estratégica en la institución educativa, donde el talento humano y su desempeño poseen un alto nivel de importancia a la hora de las mejoras organizacionales.

Palabras clave: calidad educativa, análisis estructural, talento humano.

\footnotetext{
* Licenciada en Educación mención Orientación, por la Universidad del Zulia (LUZ). Magíster en Orientación mención Laboral (LUZ). Miembro del Programa de Educación Continua del Doctorado en Ciencias Humanas (LUZ), bajo la línea de investigación: sistemas humanos. Correo electrónico: milvelasquez@gmail.com
} 
Use of the Prospective Structural Analysis to Characterize Variables of Educational Quality in Primary and Secondary Venezuelan Institution

\section{Abstract}

The article purpose was to characterize the educational institution considering quality management. Was applied a participatory action research design, with an interpretive hermeneutical approach. The methodological description came from the prospective structural analysis, by exploring the organization starting from the planning of activities according to the educational processes, keeping the congruence with the institutional mission and vision; exploring the management and organization related with the structure and guidelines of the corporation where the institution belongs; and recognizing control of activities and staff. The Structural analysis use MICMAC as prospective strategy. Among the findings, key variables emerge from the prospective, highlighting strategic planning in the educational institution, where human talent and performance have a high level of importance in order to organizational improvements.

Keywords: Educational quality, structural analysis, human talent.

Uso da análise estrutural prospectiva para caracterizar variáveis de qualidade educacional na instituiçáo primária e secundária venezuelana

\section{Resumo}

O objetivo do artigo foi caracterizar a instituição de ensino considerando a gestão da qualidade. Foi aplicado um projeto de pesquisa-açáo participativa, com abordagem hermenêutica interpretativa. A descrição metodológica veio da análise estrutural prospectiva, explorando a organização a partir do planejamento das atividades de acordo com os processos educacionais, mantendo a congruência com a missão e a visão institucional; explorar a gestão e organização relacionadas à estrutura e às diretrizes da corporação à qual a instituição pertence; e reconhecendo o controle das atividades e da equipe. A análise estrutural usa o MICMAC como estratégia prospectiva. Dentre os achados, variáveis-chave emergem do prospectivo, destacando o planejamento estratégico na instituição educacional, onde o talento e o desempenho humanos têm alto nível de importância para melhorias organizacionais.

Palavras-chave: qualidade educacional, análise estrutural, talento humano. 


\section{INTRODUCCIÓN}

La calidad debe formar parte de la práctica de los principios de los individuos en todos los ámbitos de la vida cotidiana, pues de allí depende el éxito de un país, organización o familia, siendo la misma prioridad para los hombres y mujeres que desean el bien para ellos mismos y la humanidad, con la finalidad de ofrecer un servicio de mejoras ilimitadas. En el aspecto institucional, el establecimiento de la calidad permite con ello que el cliente se sienta complacido y valorado, mejorando, por ende, toda práctica asociada al desarrollo humano.

Según lo antes mencionado, podemos decir que la calidad debe asumirse como un principio inherente a la actividad humana y acciones sociales debido a que, como un sistema humano, la calidad da como resultado una excelente proyección y distinción de las acciones propias de una organización, empresa, institución o persona.

Asimismo, es importante establecer que la calidad es un aspecto de constante evaluación para el crecimiento de los sistemas, razón por la cual existe diversos medios de estudiarle y desarrollarla en las organizaciones

En el presente documento, se desarrollan los elementos asociados a la calidad, propuestos como estudio de caso en la Unidad Educativa Autónoma (UEA) Nicolás Curiel Coutinho. Para tal fin, se realiza la descripción de los elementos asociados a la calidad de la institución: todo a luz del uso de la Matriz de Impactos Cruzados Multiplicación Aplicada a una Clasificación (MICMAC) como método de análisis estructural.

Lo anterior surge de reconocer el análisis estructural prospectivo como una herramienta de mejoramiento en todo aspecto de las ciencias sociales. Siguiendo a Arango y Cuevas (2014), «el análisis prospectivo permite que las ciencias sociales puedan identificar futuros peligros y visualizar posibles oportunidades, así como establecer una variedad de enfoques posibles para la resolución de problemas» (p. 1).

De esta manera, el material se divide estableciendo el marco teórico desde la descripción del análisis estructural prospectivo propuesto por Michel Godet (1979, citado por Arcade, Godet, Meunier y Roubelat, 2004), en la década de 1970. La relevancia de este modelo es la revisión anticipatoria de las características institucionales en camino a establecer y ejecutar un plan estratégico que favorezca la planificación a futuro de la organización. Este documento se centra en describir y establecer las variables claves relacionadas a la calidad educativa de la institución educativa de primaria y secundaria Nicolás Curiel Coutinho, llevando paso a paso el análisis estructural por medio de una de las 
herramientas propuestas por Godet (2007), la «Matriz de Impactos Cruzados Multiplicación Aplicada a una Clasificación», en adelante MICMAC.

Siguiendo las ideas del Instituto de Prospectiva Estratégica de Godet (2007), la prospectiva permite explorar las posibles y/o probables evoluciones futuras de empresas, grupos, sectores, organizaciones, instituciones, temáticas o problemáticas de toda índole (políticas, económicas, tecnológicas, sociológicas, etc.) a medio y largo plazo, mediante el análisis de las variables que más influirán en la evolución, y teniendo en cuenta los comportamientos de los actores implicados (Guzmán, Malaver y Rivera, 2005, citado por Arango y Cuevas, 2014).

Los pasos de este análisis se dividen y explican en este material desde la metodología, siguiendo los pasos que establecen resultados que permitan su discusión y respectivas conclusiones, así como las recomendaciones sobre las acciones (prospectiva o futuro) de la organización, la UEA Nicolás Curiel Coutinho, en relación con la gestión de calidad educativa.

\section{MarCo TEÓRICO}

Para establecer la calidad educativa, es necesario el diagnóstico estratégico institucional, siendo importante abordar elementos esenciales dentro del análisis estructural prospectivo, lo cual va a permitir establecer las variables esenciales o la caracterización del sistema organizacional de acuerdo a la calidad educativa.

De acuerdo con Arango y Cueva (2014), la prospectiva es entendida como un análisis que se realiza a futuro y que busca identificar cuál será el impacto que ejercerán ciertas variables en determinado fenómeno. Este enfoque fue aplicado a mediado de la década de 1970 por Michel Godet (1979 citado por Arcade et al., 2004), siguiendo los estudios de Jay Forrester, y considerado como estrategia para alcanzar los objetivos y prever problemáticas posibles en cualquier sistema.

Godet (2007) establece y describe diversas etapas y herramientas para el análisis. En este documento, se utiliza el MICMAC o Matriz de Impactos Cruzados Multiplicación Aplicada a una Clasificación que establece variables claves y su posible influencia entre ellas (impacto).

En palabras de Godet (2007) el análisis estructural es una herramienta de estructuración de una reflexión colectiva. Ofrece la posibilidad de describir un sistema con ayuda de una matriz que relaciona todos sus elementos constitutivos. Esta herramienta permite vincular las características de la organización a la luz de personas dentro de la misma (consejeros o informantes claves) y de la descripción inicial que permite tomar el contexto de la misma (descripción de la UEA Nicolás Curiel Coutinho). 
El análisis estructural comprende tres etapas:

I. Inventario de variables

II. Descripción de las relaciones entre variables (uso de la matriz de impacto cruzado)

III.Identificación de variables esenciales (determinación del tipo de variable en el sistema)

Estas variables, descritas a profundidad a la hora de establecer los resultados junto a la matriz MICMAC, corresponden a lo siguiente:

a) Variables determinantes o «influyentes»: son variables muy influyentes y un tanto dependientes. La mayor parte del sistema depende de estas variables. Son las variables más importantes, ya que pueden actuar sobre el sistema dependiendo de cuanto podamos controlarlas.

b) Variables relé: son muy influyentes y muy dependientes al mismo tiempo. Cualquier acción sobre ellas tiene consecuencias sobre las otras variables, pero estas consecuencias pueden tener un efecto boomerang que amplifica o bien detiene el impulso inicial. Este grupo a su vez se divide en dos tipos:

i. Variables de riesgo: son las variables que tienen muchas oportunidades de despertar el deseo de actores importantes, ya que, dado su carácter inestable, son un punto de ruptura para el sistema.

ii. Variables blanco: son más dependientes que influyentes por lo que se pueden considerar como el resultado de la evolución del sistema. Sin embargo, es posible actuar deliberadamente sobre ellas para que evolucione de la forma deseada. Estas variables representan posibles objetivos para el sistema en su totalidad.

c) Variables autónomas o excluidas: son poco influyentes y poco dependientes. Por un lado, no detienen la evolución del sistema, pero tampoco permiten obtener ninguna ventaja del mismo.

d) Variables dependientes o de resultado: son especialmente sensibles a la evolución de las variables influyentes y/o las variables relé. Son variables de salida del sistema.

e) Variables reguladoras: pueden actuar sucesivamente como variables secundarias, débiles objetivos y variables secundarias de riesgo.

Y se ubican a nivel de análisis de la siguiente manera: 
Figura 1. Denominación y relación de variables de acuerdo a la herramienta MICMAC

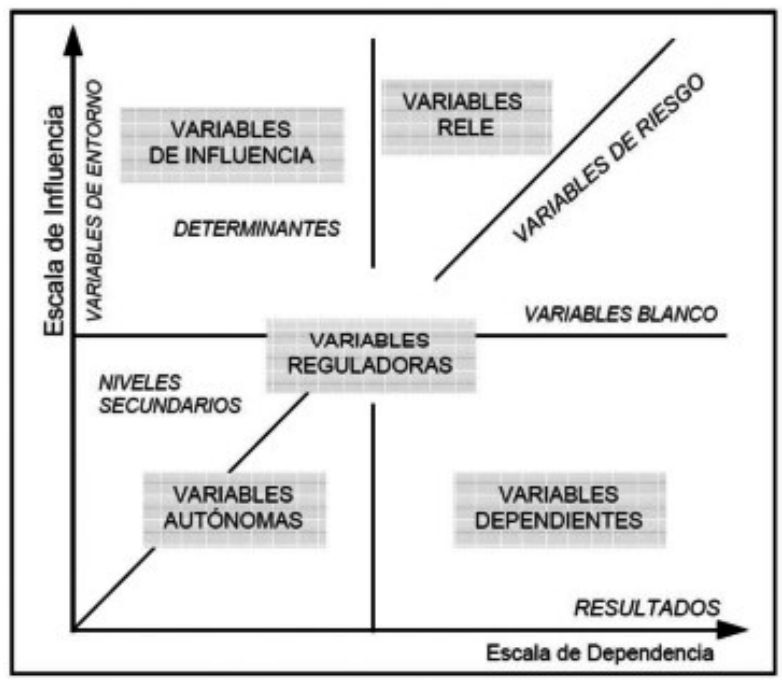

Para hacer uso de los pasos del análisis estructural, se combina la información documental y los datos de los actores implicados, a fin de establecer la siguiente descripción organizacional.

\subsection{Descripción de la organización}

La UEA Nicolás Curiel Coutinho (NCC) es un colegio con una calidad educativa que ha permanecido a lo largo de sus 72 años, manteniéndose bajo las líneas y directrices de la compañía estatal petrolera de Venezuela, Petróleos de Venezuela, Sociedad Anónima (PDVSA) y Ministerio del Poder Popular para la Educación de Venezuela (MPPE). Por lo antes señalado, se entiende que el personal de la institución se rige bajo la normativa descrita por PDVSA, en conjunto con su Federación Unitaria de Trabajadores y Trabajadoras del Petróleo, del Gas, sus Similares y Derivados de Venezuela (FUTPV) y que se encuentra expresa en su Convención Colectiva de Trabajo para el periodo 2015-2107 (2015); lo que describe a los docentes como trabajadores del estado venezolano que a su vez reciben y cumples las pautas a nivel educativo provistas por el MPPE.

En dicho sentido, esta institución pertenece al Estado, pero solo asila a estudiantes y familiares de trabajadores petroleros regidos por el contrato colectivo cláusula diecisiete y dieciocho. Enseñanza para hijos, hermanos nietos y sobrinos (PDVSA, 2015, p. 18), la cual indica que la empresa aceptará 
gratuitamente en sus institutos de educación preescolar, básica y media diversificada a los parientes cercanos de sus trabajadores, garantizando el desarrollo de las actividades deportivas y culturales, el cual se encargará de dotar a las mismas con recursos pedagógico y talento humano especializado para optimizar una educación de calidad.

Cuando hablamos de compromiso y excelencia, hacemos énfasis del lema educativo que guarda consigo la NCC, ya que es un colegio que se proyecta bajo los estándares de la tradición, lo que la ha acreditado como patrimonio cultural de la comunidad y está caracterizada por ser una de las organizaciones educativas en la búsqueda constante de mejoras en las demandas del sistema. Este colegio se encuentra ubicado en Venezuela, Estado Falcón y Municipio Carirubana, en la ciudad de Punto Fijo, específicamente en la avenida 14 (entre la calle 4 y 5 de la Comunidad Cardón).

En relación con el personal que labora en la institución, se encuentra constituido de la siguiente manera: Una líder de escuelas, que está encargada de gestionar los recursos dirigidos a la UEA Simón Bolívar y UEA Nicolás Curiel Coutinho, le rinde cuentas al gerente de RRHH del Centro de Refinación Paraguaná y a ella le debe reportar la directora, la cual responde a la máxima autoridad dentro de la NCC. Posteriormente, a ella le reportan los subdirectores de la escuela, (tres académicos y uno administrativo), así como los coordinadores (operativos y de protección y desarrollo estudiantil) y asesores de áreas (cultura, idiomas, matemática, ciencias y deportes).

En cuanto a los subdirectores académicos, se debe considerar que poseen una masa pequeña de trabajadores a su cargo, conformado por los coordinadores operativos de las etapas (Educación Inicial, Básica y Media general), y a ellos le responden los coordinadores operativos, por etapas, que están a cargo de ejercer función supervisoras a 114 docentes constituidos entre especialistas, maestros de Educación Básica Integral, profesores de Educación Inicial, auxiliares y docentes de Labor Casual.

En relación con esta estructura organizacional, los docentes y auxiliares son considerados subalternos de los coordinadores de las etapas y los especialistas de las áreas: electricidad, geografía, física, historia, música, inglés, castellano, deporte, química y biología, por lo que les reportan sus trabajos administrativos y pedagógicos a los coordinadores del área. Cabe destacar que el personal especialista docente rotativo, psicopedagogos, orientadores y psicólogos están sujetos a la coordinadora de Protección y Desarrollo Estudiantil (Coprode).

La UEA Nicolás Curiel Coutinho, por medio de su organización de trabajo, exige que los gerentes, líderes, docentes, y comunidad educativa, en general, pongan en práctica su rol contralor y mediador de la institución 
para responder de manera eficiente a la dinámica educativa, atendiendo así a las exigencias del colectivo. Es por ello que, para mantener la calidad educativa en la NCC y su realidad social, se le hace seguimiento anual al Proyecto Pedagógico Integral Comunitario, el cual a su vez emite el diagnóstico para atacar las posibles amenazas y debilidades que padece dicho colegio, dando respuesta a la transformación curricular y las orientaciones emanadas por el MPPE, la cual nos invita a integrar escuela-comunidad a efecto de garantizar un aprendizaje de calidad a los niños, niñas y adolescentes que egresan de esta institución, bajo la acción educativa de la filosofía constructivista.

Lo anterior toma en cuenta la Ley Orgánica de Educación (Asamblea Nacional de Venezuela, 2009), Ley de Protección del Niño, Niña y Adolescente (Asamblea Nacional, 2000), Ley para Personas con Discapacidad (Asamblea Nacional, 2007) y la Constitución Nacional de la República Bolivariana de Venezuela (Asamblea Nacional Constituyente, 2000), con la intención de continuar fortaleciendo desde la interdisciplinariedad hacia la transdisciplinariedad dinamizada a través de los proyectos de aprendizaje.

En cuanto al análisis interno del PEIC (2017-2018), se determina la misión y visión de la UEA Nicolás Curiel Coutinho, cuya MISIÓN es la siguiente:

Ser reconocidos como la Institución Educativa Autónoma que logre mantener criterios de calidad orientados hacia la excelencia, logrando ser reconocidos a nivel nacional e internacional, formando individuos íntegros, capaces de tomar decisiones con responsabilidad, preparados para desempeńar su rol dentro de un entorno abierto y competitivo (Comunidad Educativa UEA Nicolás Curiel Coutinho, 2017, p. 4).

Por otro lado, se destaca la visión de la organización educativa cómo:

Formar individuos de manera integral, con capacidad de adaptarse y liderar procesos de transformación de la sociedad mediante la aplicación de conocimientos adquiridos en la dinámica, enseńanza - aprendizaje, el cual debe estar enmarcado en la excelencia educativa e integración con la comunidad, resaltando los valores morales y acatando los convenios, lineamientos y políticas de PDVSA a sus trabajadores (Comunidad Educativa, 2017, p.4).

En el caso de la UEA Nicolás Curiel Coutinho, el personal se encuentra en un ambiente laboral un poco variante, observándose cambios constantes de directrices: esto se debe a las actividades y los diversos procesos a ejecutar, en relación con las diferentes normativas emitidas por entes importantes para la organización como lo son el MPPE y PDVSA.

En relación con el diagnóstico aplicado a la UEA Nicolás Curiel Coutinho, se puede evidenciar que desde las etapas de la gerencia se lleva una planificación 
de actividades acorde a los procesos educativos, cumpliendo con los parámetros establecidos en consonancia a su misión, visión y con las normativas impuestas por el Ministerio del Poder Popular para la Educación, adoptando una dirección de situaciones y organización que responde a la estructura y lineamientos de la corporación donde pertenece la institución. Adicional a ello, se observa que las acciones de control se realizan según los eventos y responsabilidades que tiene cada persona a su cargo.

\section{Metodología}

Para develar los cambios en la calidad educativa en la organización estudiada, haciendo uso del análisis estructural prospectivo de Godet (2007), se estableció la metodología de acuerdo a la intencionalidad de esta investigación, la cual persigue el determinar las características existentes en la UEA Nicolás Curiel Coutinho desde la gestión de calidad.

Siguiendo el proceso de análisis, este material se suscribe desde investigación acción participante y el enfoque hermenéutico interpretativo, partiendo del contacto con miembros de la institución (actores involucrados). De esta manera, se estableció la construcción de la investigación desde el análisis estructural y partiendo del abordaje a cinco actores que trabajan en el colegio, las cuales suministraron información valiosa y permitió establecer la descripción de la organización.

Mediante el uso del análisis estructural, para diagnosticar los elementos organizacionales existentes en la UEA Nicolás Curiel Coutinho desde la calidad, surgen los siguientes insumos, descritos anteriormente entre los pasos del análisis estructural.

\subsection{INVENTARIO DE VARIABLES}

Consta de las siguientes fases: la aproximación primaria de código; proveniente del análisis del material documental referente a la institución y el contacto inicial con los actores involucrados en la investigación. Tales códigos, luego se analizan de manera general con los actores involucrados a la investigación a fin de establecer los metacódigos de la segunda fase y continuar con el paso 2 del análisis estructural (el uso del MICMAC que está descrito en los resultados):

\section{i. Primera aproximación a códigos}

Posterior a la descripción organizacional, se establecen factores o variables relevantes a la luz de la calidad educativa en la institución. 
Tabla 1. Primera aproximación a códigos

\begin{tabular}{clrl}
\hline No & \multicolumn{1}{c}{ Códigos } & & \multicolumn{1}{c}{ Códigos } \\
\hline 1 & Compromiso y excelencia & 28 & Cambios de directrices \\
2 & Lema educativo & 29 & Preparación personal-profesional \\
3 & Tradición & 30 & Descripción de funciones y cargos \\
4 & Talento humano especializado & 31 & Talento dentro de la comunidad \\
& & & educativa \\
5 & Recursos pedagógicos & 32 & Alianzas estratégicas \\
6 & Patrimonio cultural de la & 33 & Actividades creativas e innovadoras \\
& comunidad & & \\
7 & Desarrollo de las actividades & 34 & Integración y compromiso de los \\
& & & representantes \\
8 & Organizaciones educativas en & 35 & Comunicación entre el personal \\
& constantes mejoras & & directivo-docente \\
9 & Demandas del sistema & 36 & Falta de espacios y equipos de \\
& & & computación \\
10 & Líder de escuelas & 37 & Déficit de acceso al intranet \\
11 & Gestión de los recursos & 38 & Lineamientos de contratación \\
12 & Organización de trabajo & 39 & Procesos de transformación \\
& & & curricular \\
13 & Rol contralor & 40 & Planificación de actividades \\
14 & Dinámica educativa & 41 & Procesos educativos \\
15 & Exigencias del colectivo & 42 & Dirección de situaciones y \\
& & & organización \\
16 & Calidad educativa & 43 & Cambios estratégicos de personal \\
17 & Transformación curricular & 44 & Lineamientos de la corporación \\
18 & Integración escuela-comunidad & 45 & Acciones de control \\
19 & Aprendizaje de calidad & 46 & Falta de compromiso \\
20 & Transdisciplinariedad & 47 & Desmotivación salarial \\
21 & Proyectos de aula & 48 & Deserción del talento humano \\
22 & Proyecto Pedagógico Integral & 49 & Carencia de los recursos \\
23 & Misión y visión & 50 & Reducción del presupuesto \\
24 & Criterios de calidad y excelencia & 51 & Falta de supervisión gerencial \\
25 & Valores morales & 52 & Abundantes actividades \\
26 & Dinámica, enseñanza - aprendizaje & 53 & Fallas en la comunicación \\
27 & Políticas de PDVSA & & \\
\hline & & & \\
\hline
\end{tabular}

Fuente: Elaboración propia a partir de la categorización de datos descriptivos de la institución.

A partir de los códigos primarios, se procede a interrelacionarlos a fin de crear categorías de mayor tamaño, que dentro del análisis estructural prospectivo son entendidas como metacódigos. 


\section{ii. Lista de metacódigos a partir del Inventario de variables o factores}

Tabla 2. Inventario de códigos y metacódigos

\begin{tabular}{|c|c|c|c|}
\hline Códigos & Metacódigos & Etiqueta & Descripción \\
\hline $\begin{array}{l}\text { Reducción del presupuesto } \\
\text { Lineamientos de contratación } \\
\text { Desmotivación salarial }\end{array}$ & $\begin{array}{l}\text { Recursos eco- } \\
\text { nómicos }\end{array}$ & RECO & $\begin{array}{l}\text { Hace referencia a los insumos } \\
\text { y procesos inherentes al uso y } \\
\text { aprovechamiento de recursos } \\
\text { monetarios, así como su impacto } \\
\text { en el personal. }\end{array}$ \\
\hline
\end{tabular}

\begin{tabular}{|c|c|c|c|}
\hline $\begin{array}{l}\text { Proyecto Pedagógico Integral } \\
\text { Recursos pedagógicos } \\
\text { Acciones de control }\end{array}$ & $\begin{array}{l}\text { Estándares } \\
\text { de calidad en } \\
\text { procesos peda- } \\
\text { gógicos }\end{array}$ & ЕСРP & $\begin{array}{l}\text { Los procesos organizacionales } \\
\text { relacionados con el desarrollo } \\
\text { de actividades pedagógicas de } \\
\text { calidad y cumplimiento de las } \\
\text { exigencias institucionales. }\end{array}$ \\
\hline
\end{tabular}

\begin{tabular}{|c|c|c|c|}
\hline $\begin{array}{l}\text { Dirección de situaciones y orga- } \\
\text { nización } \\
\text { Líder de escuelas } \\
\text { Gestión de los recursos }\end{array}$ & $\begin{array}{l}\text { Dirección } \\
\text { educativa }\end{array}$ & DEDU & $\begin{array}{l}\text { Autoridades de la institución } \\
\text { que se encarga de gestionar los } \\
\text { recursos para los procesos organi- } \\
\text { zacionales. }\end{array}$ \\
\hline
\end{tabular}

Carencia de los recursos

Falta de espacios y equipos de computación

Déficit de acceso al intranet

Planificación de activid
Procesos educativos
Proyectos de Aula
Transdisciplinariedad

Falta de compromiso

Valores morales
Insumos institucionales de mejora educativa

Planificación
estratégica

IIME Recursos tangibles e intangibles de la institución educativa que facilitan el cumplimiento de la calidad.

PEST Conjunto de procedimientos dentro de la institución relacionados con la planeación, organización, dirección y control.

Valores personales

VPER Cualidades personales intrínsecas que desde la moralidad evidencia el colectivo de individuos.
Compromiso y excelencia

Lema educativo

Tradición

Criterios de calidad y excelencia

Lineamientos de la corporación

Misión y visión

\begin{tabular}{|c|c|c|c|}
\hline $\begin{array}{l}\text { Alianzas estratégicas } \\
\text { Talento dentro de la comunidad } \\
\text { educativa } \\
\text { Patrimonio cultural de la comu- } \\
\text { nidad }\end{array}$ & $\begin{array}{l}\text { Insumos } \\
\text { externos a la } \\
\text { institución }\end{array}$ & IEXI & $\begin{array}{l}\text { Recursos provenientes de orga- } \\
\text { nizaciones y personal ajeno a la } \\
\text { institución educativa. }\end{array}$ \\
\hline
\end{tabular}

\section{Valores institu- cionales}

VINS Cumplimiento de las directrices, políticas e ideologías que se comparten dentro de la organización y que garantizan la integridad moral y excelencia. 


\begin{tabular}{|c|c|c|c|}
\hline Códigos & Metacódigos & Etiqueta & Descripción \\
\hline $\begin{array}{l}\text { Descripción de funciones y } \\
\text { cargos }\end{array}$ & $\begin{array}{l}\text { Estructura } \\
\text { interna de } \\
\text { organización } \\
\text { educativa }\end{array}$ & EIOE & $\begin{array}{l}\text { Caracterización de los elementos } \\
\text { internos a la institución educa- } \\
\text { tiva }\end{array}$ \\
\hline
\end{tabular}

Cambios estratégicos de personal Mejoras organi- MORG Acciones dentro de la organizaOrganizaciones educativas en zacionales constantes mejoras ción que promueven cambios positivos.

Dinámica, enseńanza-aprendizaje Productos

Actividades creativas e innovadoras

Aprendizaje de calidad

Demandas del sistema

Políticas de PDVSA

Procesos de transformación curricular

Transformación curricular

\begin{tabular}{llll}
\hline $\begin{array}{l}\text { Cambios de directrices } \\
\text { Falta de supervisión gerencial }\end{array}$ & $\begin{array}{l}\text { Variables de } \\
\text { dirección de los } \\
\text { supervisores }\end{array}$ & VDSU & $\begin{array}{l}\text { Situaciones o eventos que modi- } \\
\text { fican las posturas o líneas de } \\
\text { acción de los directivos. }\end{array}$ \\
\hline $\begin{array}{l}\text { Fallas en la comunicación } \\
\text { Comunicación entre el personal } \\
\text { directivo-docente }\end{array}$ & $\begin{array}{l}\text { Comunicación } \\
\text { organizacional }\end{array}$ & CORG & $\begin{array}{l}\text { Proceso de retroalimentación } \\
\text { efectiva y eficiente de los actores } \\
\text { de la organización. }\end{array}$ \\
\hline $\begin{array}{l}\text { Abundantes actividades } \\
\text { Organización de trabajo } \\
\text { Dinámica educativa }\end{array}$ & $\begin{array}{l}\text { Tiempo como } \\
\text { exigencia orga- } \\
\text { nizacional }\end{array}$ & TEXO & $\begin{array}{l}\text { Reconocimiento de la importan- } \\
\text { cia del tiempo para el desarrollo } \\
\text { de las acciones organizacionales } \\
\text { de calidad. }\end{array}$ \\
\hline
\end{tabular}

Integración y compromiso de los representantes

Integración escuela-comunidad de calidad

Exigencias del colectivo
PPEX Resultados de las actividades desarrolladas por los actores institucionales a nivel pedagógico y con calidad.

$\begin{array}{lll}\begin{array}{l}\text { Seguimiento a } \\ \text { las demandas }\end{array} & \text { SDSU } & \begin{array}{l}\text { Cumplimiento a las directrices } \\ \text { de los entes supervisores de la } \\ \text { de superiores }\end{array} \\ \text { institución educativa. }\end{array}$


Con esta información, se completa el primer paso de análisis, procediendo de esta manera a relacionar las variables que han surgido de estos Metacódigos, por medio de la matriz de impacto cruzado, provista por Godet (2007), haciendo uso del software prospectivo MICMAC.

\subsection{Análisis estructural de las necesidades detectadas}

El siguiente paso a nivel metodológico es someter las variables establecidas a la revisión por parte de los actores vinculados a la investigación, tomando sus respuestas y estableciendo el grado de relación entre las variables para luego vaciar la información en el software prospectivo MICMAC.

Garza y Cortez (2011) señalan que la herramienta estructural MICMAC busca analizar de manera cualitativa las relaciones entre las variables que componen un sistema dentro de una empresa, organización, sociedad o país. Cabe destacar que esta herramienta ha impactado positivamente en los trabajos investigativos de campo, permitiendo su aplicación para establecer variables de desarrollo en la institución educativa abordada.

\subsection{Descripción de las relaciones entre variables}

A efectos de desarrollar la matriz de influencia cruzada entre variables (con software MICMAC), se estableció contacto con cinco actores que trabajan en la UEA Nicolás Curiel Coutinho, las cuales suministraron información valiosa que generó grandes aportes a la recolección de datos sobre la institución. Para ello, participaron dos profesoras de educación inicial, dos de básica integral y un profesor especialista de música, ellos contribuyeron a completar el cuadro respondiendo a cada par de variables, ubicadas en las columnas y filas, si existía alguna influencia, por lo que cada participante le colocó una apreciación en números cuyo valor significaba una estimación.

Esta estimación se representa de forma numérica de la siguiente manera: el 0 era nulo; el 1 poseía una ponderación baja; el 2 era mediana; el 3, alta; y P (4) si ubicaban una relación Potencial (entre las variables).

Los datos recibidos de los actores involucrados son vaciados con la intención de generar la siguiente matriz de influencia cruzada general a continuación: 
Tabla 3. Matriz de influencia cruzada

\begin{tabular}{|c|c|c|c|c|c|c|c|c|c|c|c|c|c|c|c|c|c|c|}
\hline & $\begin{array}{l}\overrightarrow{\mathrm{D}} \\
\text { 㫚 } \\
\text { O }\end{array}$ & $\begin{array}{l}\mathrm{N} \\
\mathrm{m} \\
\mathrm{O} \\
\mathrm{p}\end{array}$ & $\begin{array}{l}\omega \\
\text { 品 } \\
\text { 学 }\end{array}$ & $\begin{array}{l}A \\
\bar{z} \\
\bar{m}\end{array}$ & 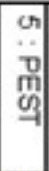 & $\begin{array}{l}\infty \\
\text { 苟 } \\
\text { 员 }\end{array}$ & $\begin{array}{l}\checkmark \\
\vdots \\
\text { ळ }\end{array}$ & $\begin{array}{l}\infty \\
\cdots \\
\underline{\mathbf{T}} \\
\underline{-}\end{array}$ & $\begin{array}{l}\emptyset \\
\cdots \\
\mathrm{m} \\
\mathrm{m}\end{array}$ & $\begin{array}{l}\overrightarrow{0} \\
3 \\
\vdots \\
0 \\
0 \\
0\end{array}$ & $\begin{array}{l}\overrightarrow{\vec{x}} \\
\ddot{0} \\
\mathrm{0} \\
\mathrm{m} \\
\times\end{array}$ & $\begin{array}{l}\vec{N} \\
\ddot{g} \\
\mathscr{C} \\
\end{array}$ & 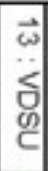 & 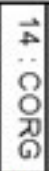 & $\begin{array}{l}\overrightarrow{\mathrm{r}} \\
\overrightarrow{\mathrm{T}} \\
\text { ठे }\end{array}$ & 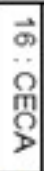 & $\begin{array}{l}\vec{v} \\
\ddot{g} \\
\bigcirc \\
\supset\end{array}$ & $\begin{array}{l}\overrightarrow{0} \\
\overrightarrow{1} \\
\underline{\mathbf{c}} \\
\underline{\underline{\zeta}}\end{array}$ \\
\hline 1: RECO & 0 & P & 3 & P & 3 & 2 & 1 & 2 & 3 & P & P & 2 & 1 & 2 & 2 & 2 & P & P \\
\hline 2: ECPP & 3 & 0 & 0 & 3 & $\mathrm{P}$ & 3 & 2 & 2 & 3 & $\mathrm{P}$ & $\mathrm{P}$ & 3 & 3 & 2 & 2 & $\mathrm{P}$ & 2 & 3 \\
\hline 3: DEDU & 1 & 3 & 0 & $\mathrm{P}$ & 3 & 3 & 2 & 1 & 3 & 3 & 3 & 2 & 2 & 3 & 3 & 2 & 3 & 3 \\
\hline 4:IIME & 3 & 2 & 2 & 0 & 3 & 1 & 3 & 0 & 0 & 3 & $\mathrm{P}$ & 3 & 2 & 3 & $\mathrm{P}$ & 2 & 1 & 1 \\
\hline 5:PEST & $\mathrm{P}$ & 3 & 3 & 3 & 0 & $\mathrm{P}$ & 3 & 3 & 3 & 3 & 3 & 2 & 3 & 2 & 3 & 3 & $P$ & 3 \\
\hline 6:VPER & 3 & 3 & 3 & 2 & 3 & 0 & 2 & 1 & 3 & 3 & 3 & 2 & 3 & 2 & 1 & 1 & $\mathrm{P}$ & 3 \\
\hline $7:$ VINS & 2 & 3 & 3 & 1 & 2 & 1 & 0 & 1 & $\mathrm{P}$ & 3 & 3 & 2 & 1 & 3 & 3 & 2 & 3 & 3 \\
\hline 8: IEXI & 1 & 3 & 2 & 1 & 3 & 1 & 2 & 0 & 1 & 2 & 3 & 3 & 2 & 3 & 1 & 1 & 2 & $\mathrm{P}$ \\
\hline 9: EIOE & 3 & 3 & 3 & 0 & 2 & 1 & 3 & 1 & 0 & P & 2 & 1 & 1 & 2 & 1 & 1 & 3 & 3 \\
\hline $10:$ MORG & 3 & $\mathrm{P}$ & 3 & 2 & $\mathrm{P}$ & 3 & 3 & 3 & 3 & 0 & $\mathrm{P}$ & 2 & 2 & 3 & 3 & 0 & 3 & 3 \\
\hline 11: PPEX & 2 & 3 & 3 & 1 & $P$ & 3 & 3 & 3 & 3 & 3 & 0 & 3 & 3 & 3 & 3 & 2 & 3 & 3 \\
\hline $12:$ SDSU & $\mathrm{P}$ & 2 & 2 & 3 & 2 & 2 & 3 & 3 & 3 & 2 & 2 & 0 & 3 & 3 & 3 & 2 & 3 & 2 \\
\hline 13:VDSU & 3 & 3 & 2 & 3 & $\mathrm{P}$ & 3 & 2 & 2 & 1 & 3 & 3 & 2 & 0 & 3 & 3 & 1 & 3 & 3 \\
\hline $14:$ CORG & 0 & 3 & 3 & 2 & 3 & $\mathrm{P}$ & 2 & 3 & 3 & $\mathrm{P}$ & $\mathrm{P}$ & 3 & 2 & 0 & 1 & 1 & 3 & 3 \\
\hline 15:TEXO & 3 & $\mathrm{P}$ & 2 & 1 & $\mathrm{P}$ & 2 & 2 & 1 & 1 & 3 & 3 & 3 & 3 & 1 & 0 & 0 & 3 & 3 \\
\hline $16: \mathrm{CECA}$ & 2 & 3 & 1 & 1 & 0 & 0 & 0 & 1 & 1 & 3 & 2 & 1 & 1 & 0 & 1 & 0 & 2 & 2 \\
\hline $17:$ DDCA & 3 & 3 & 3 & 2 & 3 & 3 & 3 & 0 & 1 & 3 & 3 & 3 & 3 & 3 & 2 & 0 & 0 & $\mathrm{P}$ \\
\hline $18:$ THUM & 3 & 3 & 3 & 1 & 3 & 3 & 3 & 2 & 3 & 3 & 3 & 3 & 3 & 3 & 3 & 1 & 3 & 0 \\
\hline
\end{tabular}

Fuente: Elaboración propia a partir de Software Prospectivo MICMAC.

El material anterior muestra la relación establecida entre las variables, todas de acuerdo al impacto de una variable sobre otra; como es percibida por los actores. Las influencias se puntúan de 0 a 3, con la posibilidad de señalar las influencias potenciales (con la letra $\mathrm{P}$ ):

0 : Sin influencia

1: Débil

2: Media

3: Fuerte

P: Potencial

Con esta matriz general de influencia cruzada, surgen dos gráficos de análisis estructural que permiten visualizar de mejor manera los resultados del estudio. 


\section{Resultados}

La aplicación de la prospectiva, como método metodológico, proporciona una serie de matrices y elementos descriptivos dentro de la organización y sistema humano estudiado. A efecto de establecer los aspectos estructurales relacionados con la calidad educativa en la institución, se revisan las gráficas de influencia o dependencia entre las variables.

De esta manera se visualizan las variables y cómo interactúan entre sí, por medio del plano de influencia directa.

Figura 2. Plano de influencia directa entre variables

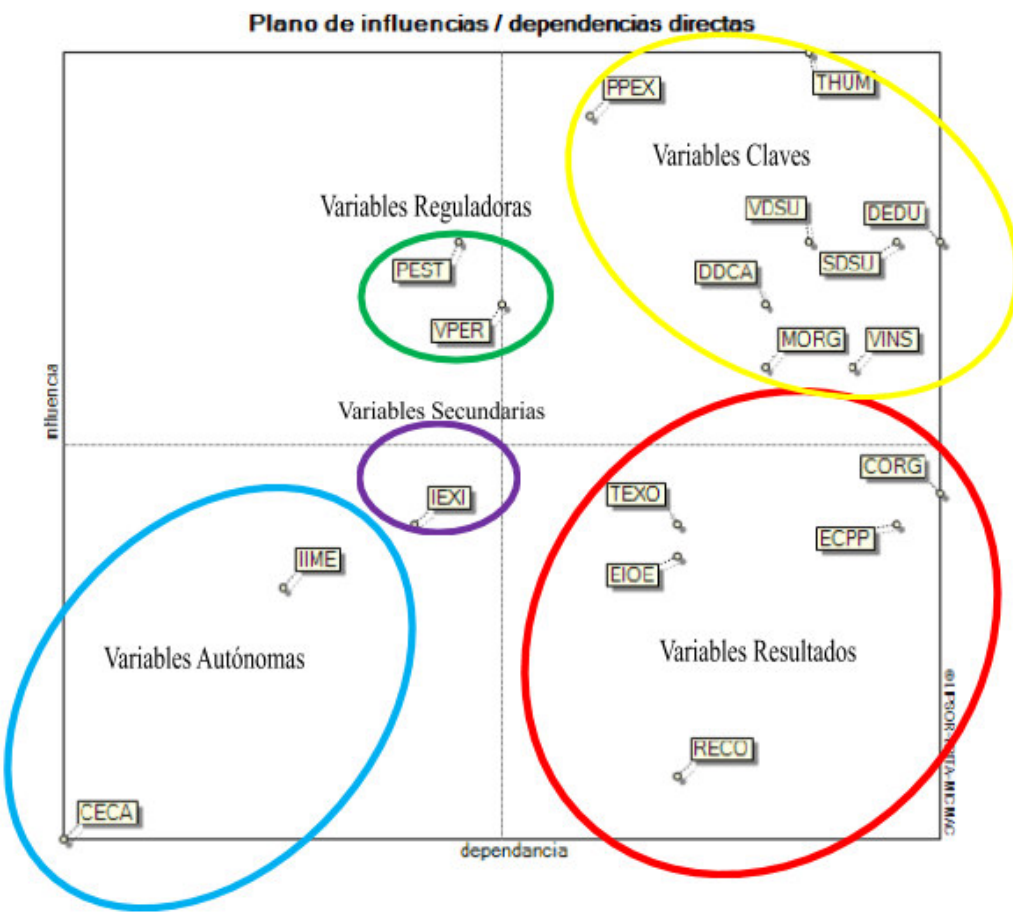

Fuente: Elaboración propia a partir de Software Prospectivo MICMAC.

Leyenda

\begin{tabular}{|l|l|}
\hline & Variables secundarias \\
\hline & Variables claves en zona de conflicto \\
\hline & Variable resultado en zona de salida \\
\hline & Variables reguladoras o palancas secundarias \\
\hline & Variables en zona de problemas autónomos \\
\hline
\end{tabular}




\subsection{Identificación de variables esenciales}

Tomando en cuenta el gráfico de influencia directa y potencial se establece las variables las cuales caracterizan los problemas de conflicto de una organización. Garza y Cortez (2011) describen las variables de la siguiente forma:

i. Variables determinantes: se encuentran en la zona superior izquierda del plano de influencia y dependencia. Son las variables que, según su evolución a lo largo del periodo de estudio, se convierten en frenos o motores del sistema. Estas pudieran ser propulsoras o inhibidoras del sistema. El objetivo es que sean propulsoras y determinen las conductas adecuadas del sistema.

ii. Variables entorno: son las variables con escasa dependencia del sistema y pueden ser consideradas un decorado del sistema. Se encuentran en la zona media de la parte izquierda del plano de influencia y dependencia. El objetivo es complementar su valor agregado al sistema.

iii. Variables reguladoras: son las variables situadas en la zona central del plano de influencia y dependencia. Se convierten en llave de paso para alcanzar el cumplimiento de las variables clave. Determinan el buen funcionamiento del sistema en condiciones normales. Se sugiere evaluar de manera consistente y con frecuencia periódica estas variables.

iv. Palancas secundarias: son las variables complementarias a las variables reguladoras. Actuar sobre ellas significa hacer evolucionar las variables reguladoras que a su vez afectan a la evolución de las variables clave. En el plano de influencia y dependencia, se encuentran ubicadas debajo de las reguladoras.

v. Variables objetivo: son las variables que se ubican en la parte central a la derecha en el plano de influencia y dependencia. Son muy dependientes y medianamente motrices, de ahí su carácter de tratamiento como objetivos, puesto que en ellas se puede influir para que su evolución sea aquella que se desea

vi. Variables clave: son las variables que se encuentran en la zona superior derecha del plano de influencia y dependencia, también nombradas variables reto del sistema. Son muy motrices y muy dependientes, y perturban el funcionamiento normal del sistema. Estas variables determinan el propio sistema. Son, por naturaleza, inestables y se corresponden con los retos del sistema. En resumen, deben continuamente 
tener retos que propicien el cambio del sistema a un nivel más óptimo. Son las que mantienen lubricado el sistema.

vii. Variables resultado: son variables que se caracterizan por su baja motricidad y alta dependencia. Se encuentran en la zona inferior derecha del plano de influencia y dependencia, y suelen estar junto con las variables objetivo, indicadores descriptivos de la evolución del sistema. Se trata de variables que no se pueden abordar de frente sino a través de las que dependen en el sistema. Estas variables requieren un seguimiento y monitoreo estrecho que permita verificar la efectividad del sistema en general.

viii. Variables autónomas: son variables poco influyentes o motrices y poco dependientes, se corresponden con tendencias pasadas o inercias del sistema o bien están desconectadas de ese. En el plano de influencia y dependencia, se encuentran en la zona inferior izquierda. No constituyen parte determinante para el futuro del sistema. Se constata frecuentemente un gran número de acciones de comunicación alrededor de estas variables que no constituyen un reto. Hay que alinear estas variables a la estrategia Lean de la empresa. Se sugiere dar más valor a esas variables. En este momento, son poco influyentes en el sistema. (Garza y Cortez, 2011, pp. 341- 342)

De acuerdo a las definiciones anteriores, en la institución educativa UEA Nicolás Curiel Coutinho, podemos apreciar que las variables en la zona de conflicto son el talento humano (THUM), variables de dirección de los supervisores (VDSU), dirección educativa (DEDU), productos pedagógicos de excelencia (PPEX), seguimiento a las demandas de superiores (SDSU), valores institucionales (VINS), desempeño docente de calidad (DDCA), mejoras organizacionales (MORG).

Adicional a esto, encontramos variables reguladoras: los valores personales (VPER) e insumos externos de la institución (IEXI), la cual se conjuga con la planificación estratégica (PEST), como palancas secundarias. En cuanto a las variables resultado: recursos económicos (RECO), el tiempo como estrategia organizacional (TEXO), estructura interna de las organizaciones educativas (EIOE), estándares de calidad en los procesos pedagógicos (ECPP), comunicación organizacional (CORG). Por otro lado, observamos las variables autónomas: insumos institucionales de mejoras educativas (IIME) y la comunidad como evaluador de calidad (CECA). 


\section{Discusión}

Los resultados descritos previamente muestran la realidad organización y, a su vez, permiten un estudio a profundidad de las variables, con el propósito de establecer posteriores planes de acción acordes a la realidad reflejada.

En este caso, las variables interactúan entre sí, explicando el funcionamiento de los grupos humanos de la institución, grupo docente con recursos para desarrollar ampliamente sus actividades con altos criterios de calidad. Sin embargo, se encuentran también con en el campo de responder a las demandas de los supervisores para procurar productos pedagógicos y cumplimiento de la planificación estratégica de la institución. Y se refleja también, en el plano, el papel que se les otorga a los valores personales para poder cumplir con los compromisos adquiridos en el sistema de trabajo.

\subsection{Influencias en el sistema}

Siguiendo la descripción de variables y su ubicación en el plano de influencia, se presenta adicional el gráfico de influencia directa, a fin de establecer las implicaciones entre variables con mayor influencia en el sistema.

Figura 3. Gráfico de influencia directa

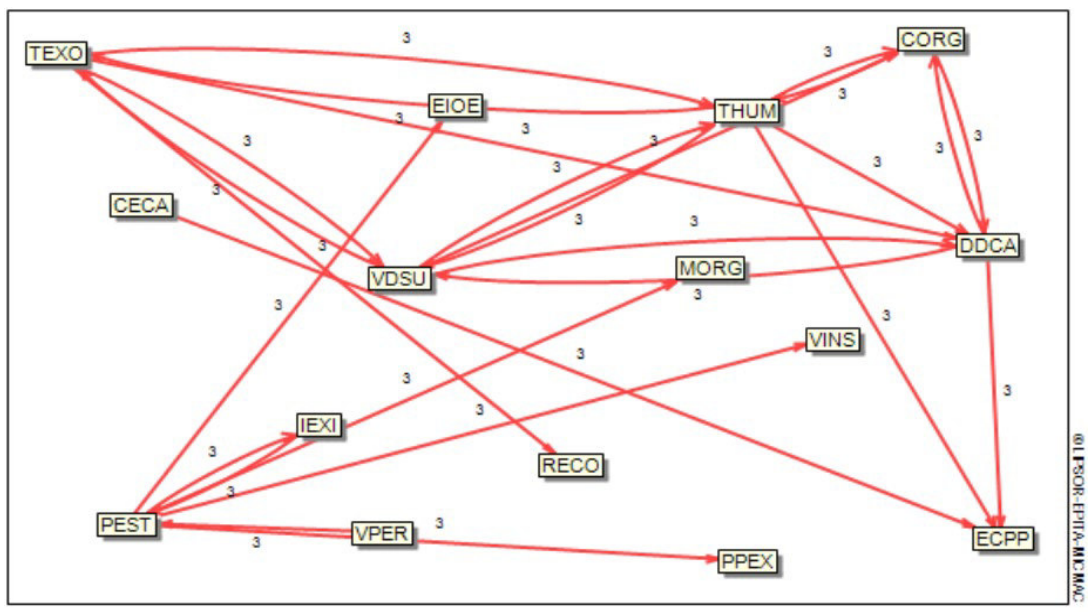

Influencias más débiles

- Influencias débiles

- Influencias medias

- Influencias relativamente importantes

- Influencias más importantes

Fuente Producto del uso del software Prospectivo MICMAC para el análisis de la Matriz de Influencia Cruzada elaborada a partir de los actores involucrados en la investigación. 
Al observar el gráfico de influencia potencial, se evidencia como punto focal el talento humano (THUM), variables de dirección de los supervisores (VDSU) y el desempeño docente de calidad (DDCA). Estas deben ser consideradas y atendidas para buscar estrategias que garanticen mejoras en la calidad educativa de la institución.

Es importante mencionar que las mismas están generando cambios constantes, propiciando que la institución educativa se vea afectada en los procesos organizacionales, poniendo en peligro el producto final que es una educación basada en valores y de excelencia.

\section{Conclusiones}

De este análisis estructural aplicado para determinar las características existentes en la UEA Nicolás Curiel Coutinho, desde la gestión de calidad, surgieron elementos a discutir. En cuanto a los estándares de calidad, esta institución educativa ha sufrido cambios que han ocasionado la disminución de la misma, muchos de los factores tanto internos como externos surgieron posterior a la aplicación de MICMAC dentro de las variables claves del sistema. Factores nombrados desde la descripción o diagnóstico inicial se reflejan al establecer conflictos institucionales desde el talento humano o personal que labora. De allí, se nombra la falta de compromiso debido a la desmotivación salarial, deserción del talento humano que se asocian a las variables de talento humano (THUM), dirección de los supervisores (VDSU), dirección educativa (DEDU), productos pedagógicos de excelencia (PPEX).

Aspectos como la falta de supervisión gerencial, fallas en la comunicación entre los miembros que la conforman se relacionan con las variables de dirección de los supervisores. De igual manera, sucede con los códigos de seguimiento a las demandas de superiores (SDSU), valores institucionales (VINS) y las mejoras organizacionales (MORG).

Siguiendo la descripción de brechas realizadas dentro del análisis estructural, se enmarcan las variables claves de esta investigación en el elemento de gran importancia y transición en una institución: Su talento humano. Es el personal de la escuela el que está mostrando las limitaciones del sistema, reflejando sus dificultades de comunicación con los supervisores. Está aunado a una ambivalencia en sus productos pedagógicos usuales que se ven afectados, según los reflejos de los informantes, por carencia de los recursos, debido a la reducción del presupuesto, abundantes actividades y poco tiempo para ejercerlas. 
De todo lo antes mencionado, surge la caracterización de la gestión de calidad educativa presente en la UEA Nicolás Curiel Coutinho. Una institución determinada por la planificación estratégica, donde el talento humano y su desempeńo mantienen un alto nivel de importancia a la hora de las mejoras organizacionales.

\section{REFERENCIAS BIBLIOGRÁFICAS}

Arcade, J., Godet, M., Meunier, F. y Roubelat, F. (2004). Análisis estructural con el método MICMAC y estrategia de los actores con el método MACTOR. Buenos Aires, Argentina.

Arango, X. y Cuevas, V. (2014). Método de análisis estructural: matriz de impactos cruzados multiplicación aplicada a una clasificación (MICMAC). Métodos y cualitativas y cuantitativas aplicables a la investigación en ciencias sociales. San Nicolás de los Garza, México: Editorial Tirant Lo Branch

Asamblea Nacional Constituyente (2000). Constitución de la República Bolivariana de Venezuela. Gaceta Oficial de la República de Venezuela No 5453. Recuperado de https://bit.ly/2wilbXc

Asamblea Nacional (2000). Ley Orgánica para la Protección de Niños Niñas y Adolescentes. Gaceta Oficial No 5. 266.

Asamblea Nacional (2007). Ley para las personas con discapacidad. Gaceta Oficial $\mathrm{N}^{\circ} 38.598$.

Asamblea Nacional de Venezuela (2009). Ley Orgánica de Educación. Gaceta Oficial de la República de Venezuela Nº 5929

Comunidad Educativa UEA Nicolás Curiel Coutinho (2017). Acuerdos de normas de convivencia escolar y comunitaria ańo lectivo 2017/2018 de la UEA Nicolás Curiel Coutinho. Documento sin publicar.

Comunidad Educativa (2017). Proyecto Pedagógico Integral Comunitario año lectivo de la UEA Nicolás Curiel Coutinho (2017-2018). Documento sin publicar.

Petróleos de Venezuela, Sociedad Anónima (2015). Convención Colectiva de Trabajo PDVSA Petróleo, S.A-FUTPV (2015-2107). Cláusula 11: Enseñanza para hijos, hermanos, nietos y sobrinos. Cláusula 12: Formación y moral del plano moral y luces (pp. 17-18).

Garza, J. y Cortez, D. (2011). El uso del método MICMAC y MACTOR análisis prospectivo en un área operativa para la búsqueda de la excelencia operativa a través del Lean Manufacturing. Innovaciones de Negocios 8(16) 335-356. Recuperado de http://eprints.uanl.mx/12560/1/A6.pdf 
Godet, M. (1979). The crisis in forecasting and the emergence of the "prospective» approach. Nueva York: Pergamon.

Godet, M (2007). La Caja de Herramientas de la prospectiva estratégica. España: Laboratorio de investigación en prospectiva estratégica, CNAM, Instituto Europeo de Prospectiva estratégica. Documento disponible en Laboratorio de Investigación en Prospectiva, Estrategia y Organización LIPSOR. Recuperado de http://www.laprospective.fr

Guzmán, A., Malaver, M. y Rivera, H. (2005). Análisis estructural. Técnica de la prospectiva. Bogotá: Universidad del Rosario. Recuperado de http://repository.urosario.edu.co/bitstream/10336/1204/1/BI\%2024.pdf 